\title{
Orbital angular momentum photonic quantum interface
}

\author{
Zhi-Yuan Zhou ${ }^{1,2 *}$, Yan Li $^{1,2}$, Dong-Sheng Ding ${ }^{1,2}$, Wei Zhang ${ }^{1,2}$, Shuai Shi ${ }^{1,2}$, Bao-Sen Shi ${ }^{1,2}$ \\ and Guang-Can Guo ${ }^{1,2}$
}

Light-carrying orbital angular momentum (OAM) has great potential in enhancing the information channel capacity in both classical and quantum optical communications. Long distance optical communication requires the wavelengths of light are situated in the low-loss communication windows, but most quantum memories currently being developed for use in a quantum repeater work at different wavelengths, so a quantum interface to bridge the wavelength gap is necessary. So far, such an interface for OAM-carried light has not been realized yet. Here, we report the first experimental realization of a quantum interface for a heralded single photon carrying OAM using a nonlinear crystal in an optical cavity. The spatial structures of input and output photons exhibit strong similarity. More importantly, single-photon coherence is preserved during up-conversion as demonstrated.

Light: Science \& Applications (2016) 5, e16019; doi:10.1038/Isa.2016.19; published online 29 January 2016

Keywords: frequency conversion; spontaneous parametric down conversion; sum frequency generation; orbital angular momentum

\section{INTRODUCTION}

Photons are very important information carriers for transferring quantum states between remote physical systems, such as atomic ensembles, ions, and solid-state systems ${ }^{1-7}$ acting as quantum memories $^{5-9}$ and quantum information processors ${ }^{10}$. Light-carrying orbital angular momentum (OAM) has stimulated considerable research interest in both classical and quantum optical fields, has exciting applications, including optical manipulation and trapping $^{11,12}$, high-precision optical measurements ${ }^{13-15}$, high-capacity free space and fiber optic communications ${ }^{16,17}$, and studies of fundamental quantum physics ${ }^{18-21}$. In quantum communication, due to the inherent infinite dimension of OAM, photons encoded in OAM space can significantly increase the information channel capability in quantum key distribution ${ }^{22-24}$. A photon in telecom band or in freespace communication window is vital to construct a long-distance high-capacity quantum communication network. So far, most quantum memories operate in the visible wavelength range ${ }^{5-9}$, only few memories can work in telecom band ${ }^{25}$. Furthermore, the signal stored is an attenuated coherent light and has the Gaussian mode. Only recently, the storage of telecom wavelength entanglement is realized in an erbium-doped optical fibre ${ }^{26}$, but the spatial mode used is Gaussian mode. Quantum memories for photons with OAM have recently been realized ${ }^{27,28}$, but all work in visible range. So a quantum interface to bridge the wavelength gap is necessary. So far, such an interface for OAM-carried light has not been realized yet.

There are some experimental realizations of quantum interfaces for single photons with Gaussian shapes ${ }^{29-38}$, either by using secondorder nonlinear processes in nonlinear crystals or by third-order nonlinear processes in atomic ensembles ${ }^{39,40}$. Frequency conversion using nonlinear crystals is much more attractive for practical applications because it can offer wide phase-matching wavelength range, in contrast to using atomic ensembles. Most of previous experiments used periodically poled $\mathrm{LiNbO}_{3}$ (PPLN) bulk crystals or waveguides to perform the frequency conversion, near unity conversion efficiency can be reached in waveguide PPLN crystals and the quantum properties of the single photons are preserved. The frequency conversion of photons with OAM using waveguide crystals is not possible because OAM modes cannot propagate in waveguides. However, our recent studies on the frequency conversion of OAM-carried light offer the possibility for realizing this aim with bulk periodically poled nonlinear crystals $^{41-43}$.

In this work, we report the first experimental realization of an OAM photonic quantum interface by up-converting a heralded OAM-carried single photons from $1560 \mathrm{~nm}$ to $525 \mathrm{~nm}$ using the cavityenhanced sum frequency generation (SFG). The conversion efficiency can reach $8 \%$ for photons carrying OAM of $1 \hbar$. We clearly demonstrate that the spatial structure of input and output photons exhibits strong similarity. We also show that the coherence properties of the single photons are retained in the conversion process. This primary study will pave the way for high-dimensional quantum information processing, creating a link between different quantum systems that work in different wavelengths by using OAM degree of freedoms of photons.

\section{MATERIALS AND METHODS}

Details of the SPDC and SFG crystals

Both the spontaneous parametric down conversion (SPDC) crystal and the SFG crystal are periodically poled potassium titanyl phosphate (PPKTP), which are manufactured by Raicol Crystals, and all of these crystals have dimensions of $1 \mathrm{~mm} \times 2 \mathrm{~mm} \times 10 \mathrm{~mm}$. The type-II

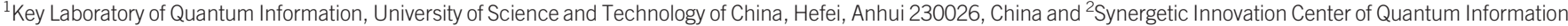
\& Quantum Physics, University of Science and Technology of China, Hefei, Anhui 230026, China

*These authors contributed equally to this work.

Correspondence: BS Shi, Email: drshi@ustc.edu.cn

Received 21 April 2015; revised 2 September 2015; accepted 4 September 2015; accepted article preview online 6 September 2015 
SPDC crystal has a poling period of $46.2 \mu \mathrm{m}$; both end faces of the crystal are anti-reflection coated for $780 \mathrm{~nm}$ and $1560 \mathrm{~nm}$, and the measured quasi-phase matching temperature of the crystal is $23.6{ }^{\circ} \mathrm{C}$. The type-I SFG crystal has a poling period of $9.375 \mu \mathrm{m}$; both end faces of the crystal are anti-reflection coated for $525 \mathrm{~nm}, 795 \mathrm{~nm}$, and 1560 $\mathrm{nm}$, and the measured quasi-phasing matching temperature of the crystal is $39.4{ }^{\circ} \mathrm{C}$. The $795 \mathrm{~nm}$ wavelength corresponding to $\mathrm{Rb}^{85} \mathrm{D} 1$ line, the $1560 \mathrm{~nm}$ is at telecom band suitable for long distance transmission. The SFG beam can be used to generate a two-color signal and idler photon source at $795 \mathrm{~nm}$ and $1560 \mathrm{~nm}$ in another crystal which has the same parameter as the SFG crystal.

\section{SFG cavity design}

The bow-tie ring cavity is designed for a single resonance at $795 \mathrm{~nm}$, and the total cavity length is $547 \mathrm{~mm}$. The input coupling mirror M1 has transmittance of $3 \%$ at $795 \mathrm{~nm}$. Mirror M2 is highly reflectively coated at $795 \mathrm{~nm}(R>99.9 \%)$, and a piezoelectric element (PZT) is attached to it to scan and lock the cavity. The two concave mirrors, M3 and M4, have curvatures of $80 \mathrm{~mm}$; M3 has a high transmittance coating for $1560 \mathrm{~nm}(T>99 \%)$ and is highly reflectively coated for $795 \mathrm{~nm}(R>99.9 \%)$, while M4 has a high transmittance coating for $525 \mathrm{~nm}(T>98 \%)$ and is highly reflectively coated for $1560 \mathrm{~nm}$ and $795 \mathrm{~nm}(R>99.9 \%)$. The fundamental cavity mode has a beam waist of $33 \mu \mathrm{m}$ at the mid-points of mirrors M3 and M4.

\section{RESULTS AND DISCUSSION}

\section{Theoretical model}

The quantum theory for SFG of continuous waves in second-order nonlinear crystals is shown as follows. Three waves are involved in the up-conversion process: one strong pump beam at frequency $\omega_{\mathrm{p}}$, one signal beam to be converted at frequency $\omega_{s}$, and the up-converted beam at frequency $\omega_{\mathrm{SFG}}$, where the frequencies of the interacting waves satisfy $\omega_{\mathrm{SFG}}=\omega_{\mathrm{p}}+\omega_{\mathrm{s}}$. The entire conversion process can be described by the following Hamiltonian ${ }^{44}$ :

$$
H_{I}=i \hbar \kappa\left(\hat{a}_{\mathrm{s}} \hat{a}_{\mathrm{SFG}}^{\dagger}-\hat{a}_{\mathrm{s}}^{\dagger} \hat{a}_{\mathrm{SFG}}\right),
$$

Here, $\hat{a}_{\mathrm{s}}$ and $\hat{a}_{\mathrm{SFG}}$ are the annihilation operators for the signal and the up-converted photons, respectively. $\kappa=g E_{\mathrm{p}}$ is a constant, where $g$ is proportional to the second-order susceptibility $\chi^{(2)} ; E_{\mathrm{p}}$ denotes the pump beam's electrical field amplitude. The Heisenberg equations of motion in the interaction picture are:

$$
\begin{gathered}
\frac{\mathrm{d} \hat{a}_{\mathrm{s}}}{\mathrm{d} t}=-\kappa \hat{a}_{\mathrm{SFG}}, \\
\frac{\mathrm{d} \hat{a}_{\mathrm{SFG}}}{\mathrm{d} t}=\kappa \hat{a}_{\mathrm{s}},
\end{gathered}
$$

The solutions to these two equations for a nonlinear interaction length $L$ are given by:

$$
\begin{gathered}
\hat{a}_{\mathrm{s}}(L)=\cos (\kappa L) \hat{a}_{\mathrm{s}}(0)-\sin (\kappa L) \hat{a}_{\mathrm{SFG}}(0), \\
\hat{a}_{\mathrm{SFG}}(L)=\sin (\kappa L) \hat{a}_{\mathrm{s}}(0)+\cos (\kappa L=\pi / 2) \hat{a}_{\mathrm{SFG}}(0),
\end{gathered}
$$

The single photon up-conversion efficiency is defined as $\eta=$ $N_{\mathrm{SFG}}(L) / N_{\mathrm{s}}(0)$, where $N_{i}=\left\langle\hat{a}_{i}^{\dagger} \hat{a}_{i}\right\rangle_{T}$ is the mean photon number in the measurement time $T ; \hat{a}_{\mathrm{s}}(0)$ and $\hat{a}_{\mathrm{SFG}}(0)$ are the annihilation operators for signal and SFG photons at 0 interaction length (at the input face of the crystal), respectively. $N_{s}(0)$ is the input signal photon number at front face of the crystal. For a practical up-converter, $N_{\mathrm{SFG}}(0)=0$, and $\eta=\sin ^{2}(\kappa L)$. The perfect conversion is achieved under the condition of $\kappa L=\pi / 2$. While the theoretical model above is for a Gaussian mode photon, it can naturally be generalized for a photon with OAM.

\section{Conversion efficiencies for different OAMs}

For frequency up-conversion using two Gaussian light beams, the quantum conversion efficiency of the signal light can be expressed as ${ }^{45}$ :

$$
\eta=\sin ^{2}\left(\frac{\pi}{2} \sqrt{\frac{P}{P_{\max }}}\right)
$$

Here, $P$ is the circulating power of the pump beam in cavity, and $P_{\max }$ is the pump power that gives unity conversion efficiency. The expression for $P_{\max }$ is:

$$
P_{\max }=\frac{\varepsilon_{0} c n_{\mathrm{s}} n_{\mathrm{SFG}} \lambda_{\mathrm{p}} \lambda_{\mathrm{s}} \lambda_{\mathrm{SFG}}}{16 \pi^{2} d_{\mathrm{eff}}^{2} \operatorname{Lh}(0, \xi)},
$$

Here, $\varepsilon_{0}$ and $c$ represent the vacuum permittivity and the speed of light in a vacuum, respectively; $n_{\mathrm{s}}$ and $n_{\mathrm{SFG}}$ are the refractive indices of the signal and up-converted beams, respectively; $\lambda_{s}, \lambda_{\mathrm{SFG}}$, and $\lambda_{\mathrm{p}}$ are the wavelengths of the three interaction waves; $d_{\mathrm{eff}}$ is the effective nonlinear coefficient; $L$ is the crystal length; and $h(\xi)$ is a parameter dependent on the focusing parameter $\xi$. Please refer to the Supplementary Information for details.

In the situation where the pump beam is in a Gaussian mode and the signal beam carries OAM, the SFG power is calculated as:

$$
P_{\mathrm{SFG}}=\frac{16 \pi^{2} d_{\mathrm{eff}}^{2} P P_{\mathrm{s}} L 2^{l}}{\varepsilon_{0} c n_{\mathrm{s}} n_{\mathrm{SFG}} \lambda_{\mathrm{SFG}}^{2} \lambda_{\mathrm{p}}} h(l, \xi),
$$

where $P_{\mathrm{s}}$ is the signal power, and $l$ is the OAM index of the signal. For $l=0$, Equation (8) is reduced to the SFG with two Gaussian beams. For more detailed derivations of Equations (7) and (8), please refer to the Supplementary Information.

\section{Up-conversion of a classical light with OAM}

To obtain an overview of frequency up-conversion of OAM-carried light, we first perform an experiment using coherent light. We want to mention the fact that demonstrations of OAM frequency conversion and conservation using classical light are also widely studied in birefringence phase matching crystals ${ }^{46-49}$. The experimental setup is shown in Figure 1a. High conversion efficiency can be achieved by placing a PPKTP crystal inside a ring cavity (please refer to materials and methods section for details of the PPKTP crystal and the cavity design). The strong pump beam is provided by a Ti:sapphire laser (Coherent, MBR110, Patrick Henry Drive Santa Clara, USA), and the light-carrying OAM by a vortex phase plate (VPP, RP Photonics, Bad Dürrheim, Germany) for conversion comes from a diode laser (Toptica, pro design, Graefelfing, Germany). The cavity is actively locked using the Hansch-Couillaud technique ${ }^{50}$. We measure the SFG power versus the signal power for various OAM with a fixed pump power of $750 \mathrm{~mW}$, the results are shown in Figure 2a. We conclude that the SFG power is linearly proportional to the input 
a

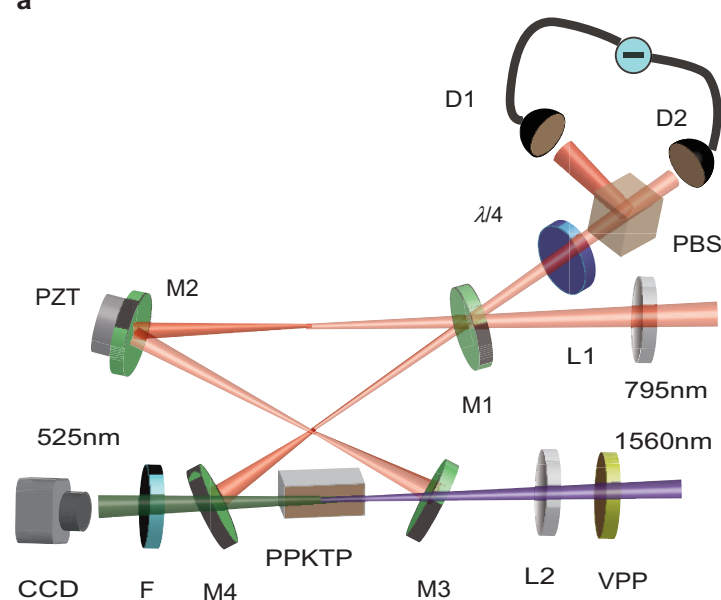

b

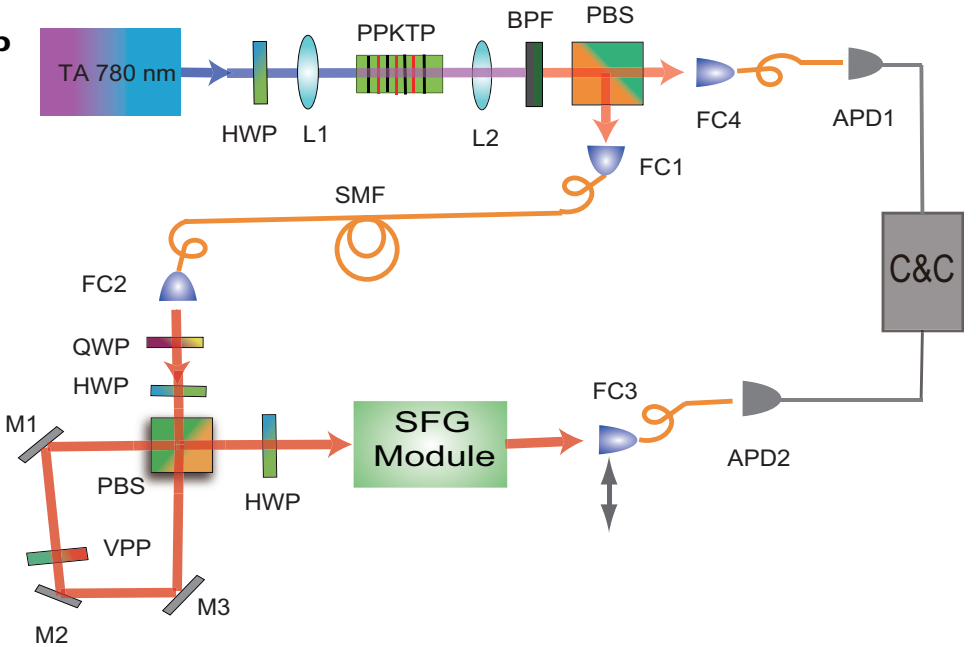

Figure 1 Setup for the cavity-enhanced up-converter module (a) and for up-converting a herald single photon with OAM (b) L1, L2: lenses; M1-M4: cavity mirrors; VPP: vortex phase plate; PBS: polarizing beam splitter; F: filters; PPKTP: periodically poled KTP crystal. HWP, QWP: half (quarter) wave plate; BPF: band pass filter; FC1-FC4: fiber couplers; SMF: single mode fiber; APD1(APD2): InGaAs (silicon) avalanche detector.

signal power for OAM values $l$ of 0,1 , and 2 ; the images inserted across the lines are the corresponding spatial shapes for the various OAM modes, and these images are acquired using a charge-coupled device (CCD) camera. The power conversion efficiencies determined using $\eta_{\text {power }}=P_{525} / P_{1560}$ are $0.66,0.259$, and 0.0893 for $l$ values of 0,1 , and 2 , respectively. The corresponding quantum conversion efficiencies defined by $\eta_{\text {quantum }}=\eta_{\text {power }} \lambda_{525} / \lambda_{1560}$ are $0.224,0.0833$, and 0.0296 , respectively. The conversion efficiencies will keep unchanged against the signal power according to the linearity of the SFG process. We also calculate the quantum conversion efficiency for different OAMs, the results are showed in Figure $2 b$, where the efficiencies are normalized with respect to the Gaussian mode. The theoretical predictions are well in agreement with experimental results. The differences in the conversion efficiencies for different OAMs are mainly caused by different overlaps between the signal and the pump beams, this can be explained by Equation (8) (for further details, please refer to the Supplementary Information). Differences in conversion efficiency for different OAM modes could be somehow compromised by pre-engineering the focus parameter and amplitude of the input signal beam.

We then test our system with attenuated coherent light. The results are shown in Figure $2 \mathrm{c}-2 \mathrm{f}$, which are obtained using a single-photon-counting camera (Andor, ICCD, Belfast, Northern Ireland) by setting it in fire-only mode, each image is accumulated with 360 frames and the background is subtracted (the dark count is 600 for each pixel in each frame), the exposure time of the ICCD is set to be $1 \mathrm{~s}$. The numbers of frames required for summation to obtain the final images shown in Figure $2 c-2 f$ are 2160, 2520, 1800 and 2520 respectively. The numbers of input photons are calibrated using an InGaAs single photon avalanche detector (APD) (Lightwave, Princeton, $30 \mathrm{MHz}$ trigger rate, 1 ns detection window, South Cranbury, USA). The recorded count rates by APD are $11.7 \mathrm{k} /$ $\mathrm{s}, 21.2 \mathrm{k} / \mathrm{s}$, and $16.8 \mathrm{k} / \mathrm{s}$ and $21.2 \mathrm{k} / \mathrm{s}$ for photons in OAM state of $|1\rangle,|2\rangle, 1 / \sqrt{2}(|1\rangle+|-1\rangle)$, and $1 / \sqrt{2}(|2\rangle+|-2\rangle)$, respectively. The actual photon number rates at the crystal's input face are $2.1 \mathrm{M} / \mathrm{s}$, 3.7 M/s, $2.8 \mathrm{M} / \mathrm{s}$, and $3.1 \mathrm{M} / \mathrm{s}$ after we consider the losses of the VPP and the input mirror M3 (the transmission efficiencies for the four input states measured with strong coherent laser beams are 0.80 , $0.79,0.74$, and 0.67 , respectively), the duty cycle of the APD
(1/33.33), and detection efficiency of 0.15 per gate. The typical donut structures can be clearly distinguished for the different OAM-carried input beams in Figure $2 \mathrm{c}$ and $2 \mathrm{~d}$, the theoretical predictions are showed in Figure $2 \mathrm{~g}-2 \mathrm{~h}$.

In addition to up-conversion of light with single OAM, we also perform up-conversion of light with OAM superposition. A modified Sagnac interferometer ${ }^{20,27,41}$ is used to generate the OAM superposition, the generated superposition state is:

$$
|\Phi\rangle_{\text {in }}=\frac{1}{\sqrt{2}}\left(|H\rangle|l\rangle+e^{i \theta}|V\rangle|-l\rangle\right),
$$

where $|H\rangle$ and $|V\rangle$ denote the polarization of the signal beam, $|l\rangle$ represents the OAM of the beam, and $\theta$ is a phase dependent on the position of the half wave plate (HWP) at the input port of the interferometer. When we insert another HWP with optical axes placed at $22.5^{\circ}$ relative to the horizontal direction, the up-converted SFG light state is (for details of the derivation, see Supplementary Information)

$$
|\Phi\rangle_{\text {out }}=\frac{1}{\sqrt{2}}\left(|l\rangle+e^{i \theta}|-l\rangle\right)|V\rangle,
$$

Equation (10) is obtained by projection 45 degree rotated two polarization components in Equation (9) onto the vertical direction, it shows that the up-converted state is a superposition of two OAMs with the same absolute value but opposite sign, the interference pattern between them has $2 l$ maximum in the azimuthal direction, similar to $2 l$ "petal." The experimental results for $l=1,2$ are shown in Figure $2 \mathrm{e}$ and $2 \mathrm{f}$, typical petals in the interference patterns show good agreement with the theoretical expectations (Figure $2 \mathrm{i}$ and $2 \mathrm{j}$ ). The mean photon number of the attenuated light at the input face of the SFG crystal in 1 ns detection window is about 0.002 , which is in the same order with a typical SPDC photon source, therefore, our system is capable of converting OAM-carried photons from SPDC.

\section{Conversion of a heralded single photon with OAM}

The photon pair is prepared by using a 780-nm laser with $120 \mathrm{~mW}$ power to pump a type-II PPKTP crystal, generating degenerate signal 

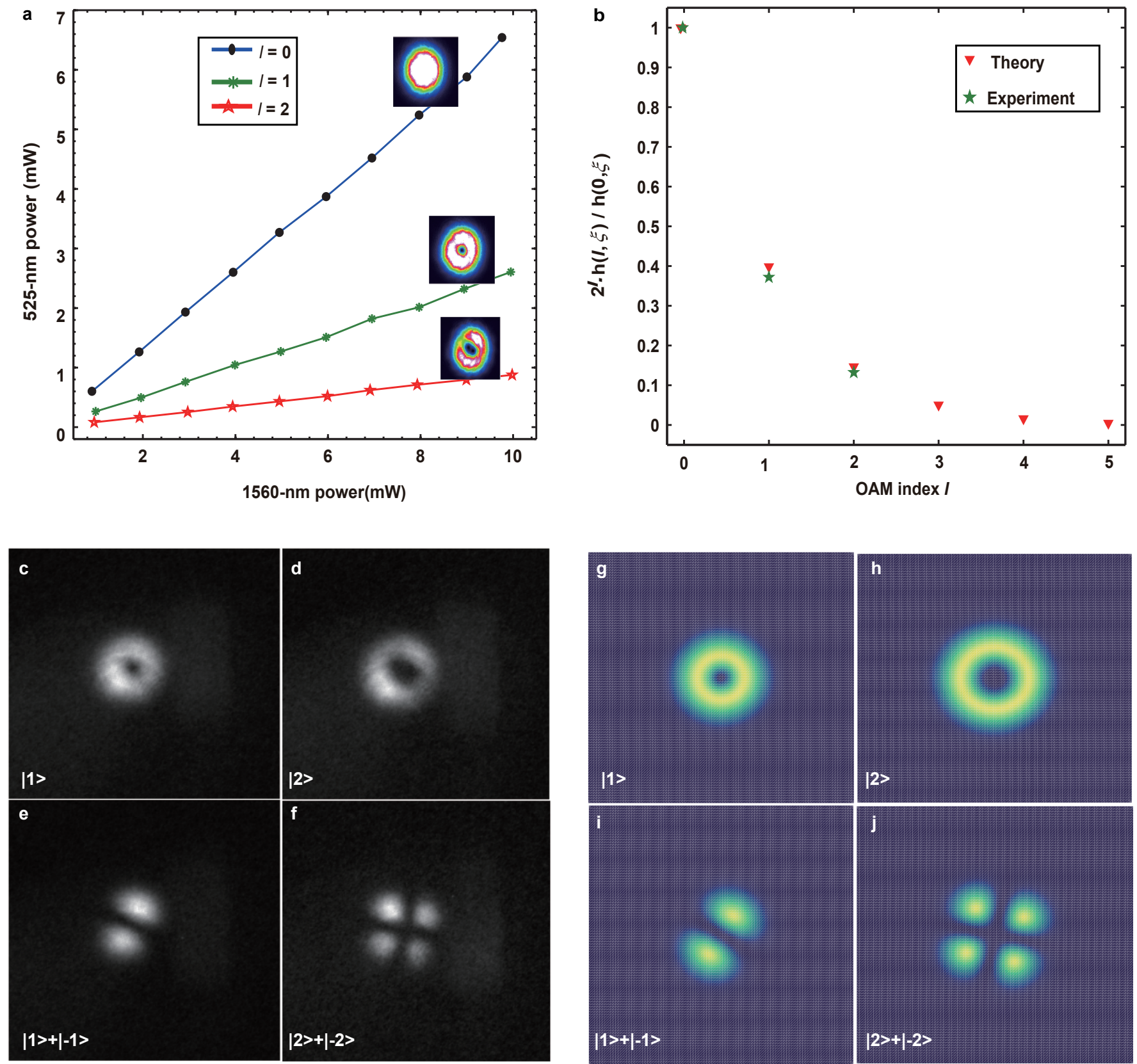

Figure 2 Experimental results with strong and attenuated coherent light at single photon level respectively. (a) The lines show the relationships between the input signal power and the SFG output powers for $I=0,1$, and 2, respectively. The inserted images across the lines are the spatial shapes for the corresponding SFG light; (b) Experimental results and theoretical simulations of up-conversion efficiency for different OAM based on Equation (8); (c)-(f) show the up-converted images of light with single OAM and superpositions input of $I=1,2$, respectively; (g)-(j) are the corresponding theoretical simulation results for (c)-(f), respectively.

and idler photons at $1560 \mathrm{~nm}$. The non-classical nature of the signal and idler photons can be characterized using the intensity cross-correlation $g_{s, i}^{(2)}$ between them ${ }^{5,51}$. The normalized second-order correlation function are defined as:

$$
g_{j k}^{(2)}(\tau)=\frac{\left\langle E_{j}^{\dagger}(t) E_{k}^{\dagger}(t+\tau) E_{k}(t+\tau) E_{j}(t)\right\rangle}{\left\langle E_{j}^{\dagger}(t) E_{j}(t)\right\rangle\left\langle E_{k}^{\dagger}(t+\tau) E_{k}(t+\tau)\right\rangle},
$$

Where indices $j, k \in\{s, i\}$ represent the signal or idler photon, respectively. The measurements of $g_{j, k}^{(2)}(\tau)$ consists of first determining the rate of coincidence detections between mode $j$ and $k$ at a time delay $\tau$. This is effectively a measurement of the non-normalized second-order coherence function, which is the numerator in Equation (11). The normalization is then performed with respect to the rate of coincidences between photons from uncorrelated pairs created at times differing by much more than the coherence time of the photons.

If we assume that the auto-correlations $1 \leq g_{s, s}^{(2)}, g_{i, i}^{(2)} \leq 2$ are satisfied, then non-classicality is provided for measured cross-correlation $g_{s, i}^{(2)}>2$. Experimentally we obtain $g_{s, i}^{(2)}=162$ between the input signal and idler photons. The crystal is described in detail in the Supplementary Information and the performance of the crystal is 

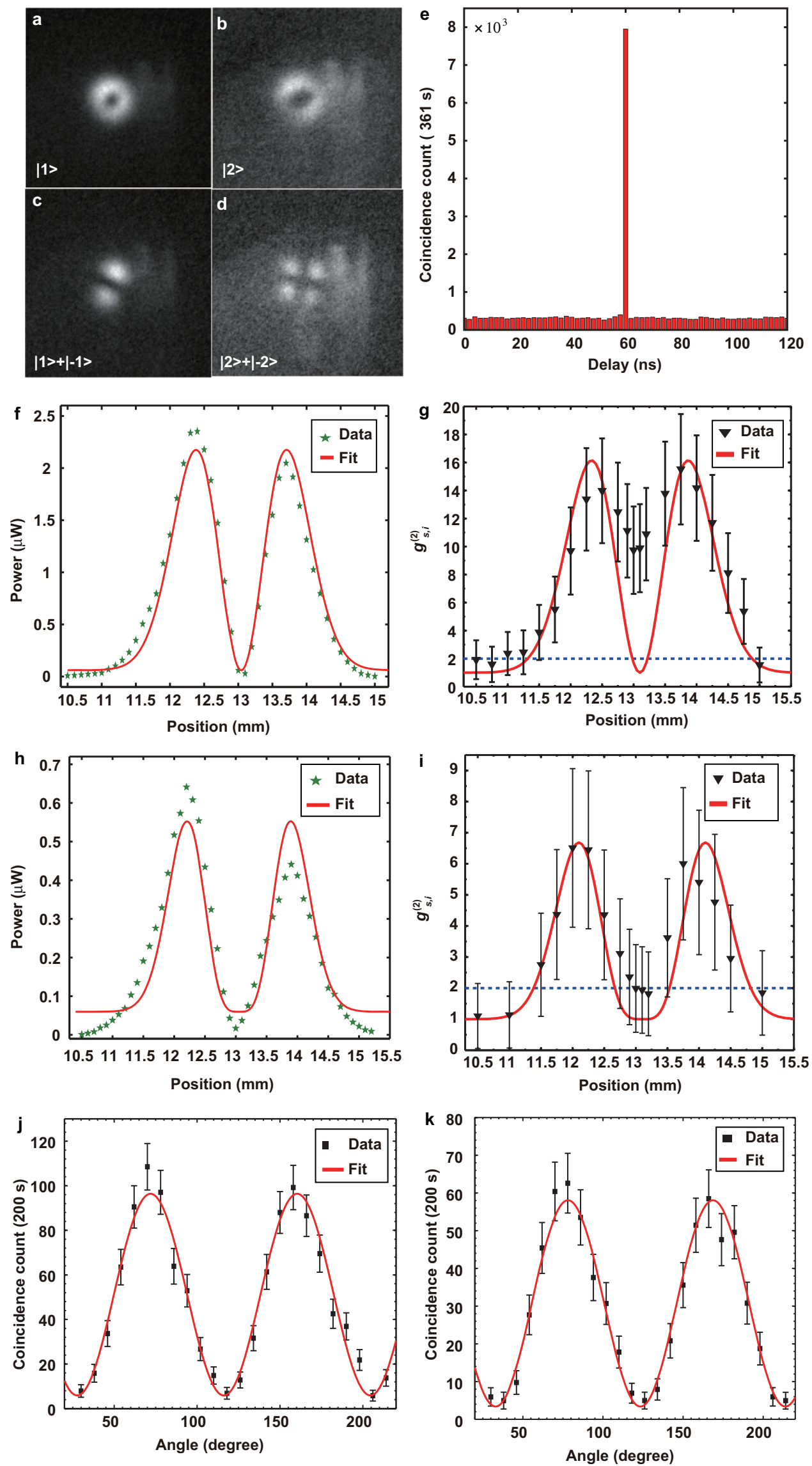
Figure 3 Experimental results for heralded single photon from SPDC (a)-(d) show SFG photon images taken using the ICCD for different input states; (e) coincidence count between idler and up-converted signal photon with the Gaussian spatial shape; (f) and (h) show one-dimensional scanning results for position-dependent power for $I=1,2$, respectively, using strong coherent pump beams; $(\mathbf{g})$ and (i) show corresponding $g_{s, i}^{(2)}$ measurement results for single photon signal inputs, error bars are estimated by assuming Poison statistics of photon measurements; ( $\mathbf{j}$ ) and $(\mathbf{k})$ show the phase-dependent coincidence counts produced by rotating the HWP for $I=1,2$, respectively.

described in our previous works ${ }^{52-54}$. The experimental setup for upconverting a herald single photon with OAM is shown in Figure 1b. We first measure the spatial structure of the up-converted photon by ICCD. The results are shown in Figure 3, where Figure 3a and 3b shows the results for the OAM states $|1\rangle$ and $|2\rangle$, and Figure $3 \mathrm{c}$ and $3 \mathrm{~d}$ shows the results for the superposition states $1 / \sqrt{2}(|1\rangle+|-1\rangle)$ and $1 / \sqrt{2}(|2\rangle+|-2\rangle)$, respectively. Images in Figure 3a-3d show that the photon with both single OAM value and the OAM superposition can be up-converted, as the typical donut shapes and interference patterns are clearly distinguished. In this experiment, the ICCD has the same settings as used in the previous experiments with the attenuated coherent light.

We also measure the cross-correlations for different input OAM states by coupling the up-converted photons into a single mode fiber (SMF). We first perform coincidence measurements between the idler and the up-converted signal photons with the input signal photon in the Gaussian mode. The results are shown in Figure 3e; The measured cross-correlation in 2 ns coincidence window is $g_{s, i}^{(2)}=25$, which demonstrates that the up-converted signal photon and the idler photon are in non-classical correlation. We then perform coincidence measurements for signal photons with OAM by scanning the SMF in the horizontal direction. The results are shown in Figure $3 \mathrm{f}-3 \mathrm{i}$. Figure $3 \mathrm{f}$ shows the result for a strong coherent input beam with $l=1$, where the position-dependent power coupling into the SMF has a dip at the center, and the theoretical fit shows good agreement with the experimental data; Figure $3 \mathrm{~g}$ shows the measured $g_{s, i}^{(2)}$ for a single photon input, where the $g_{s, i}^{(2)}$ has a dip in the raw data, and the maximum $g_{s, i}^{(2)}$ is 15.5. The deviation of the experimental data from the theoretical fit at the center is a result of the impurity of the up-converted OAM mode, which is mainly caused by misalignment and spontaneous Raman scattering noise, the noises in the up-conversion process is discussed in the Supplementary Information. Figure $3 \mathrm{~h}$ and $3 \mathrm{i}$ show the results for $l=2$, and the maximum $g_{s, i}^{(2)}$ for single photon input is 6.5. $g_{s, i}^{(2)}>2$ for $l=1,2$ are clear evidence proving the existed non-classical correlation in spatial shape between the up-converted signal and the idler photons. We should point out that the filtering of the up-conversion spatial mode using SMF introduces loss in the detection, if the spatial mode is detected directly, the value of the cross-correlation will be even larger. To show that the coherence properties are retained in the conversion process, we let the input signal photon be in the state of Equation (9), then the upconverted signal photon state is in the form of Equation (10). By filtering out a single petal of the interference pattern use a pinhole and coupling it into the SMF, we measure the coincidence dependent on the phase $\theta$ through rotating the HWP at the input port of the interferometer. This method is introduced in refs 20,27 . The filtering operation can be described using the following projection operator $\hat{P}=|\Pi\rangle\langle\Pi|$, where $|\Pi\rangle=1 / \sqrt{2}\left(|l\rangle+e^{i \varphi}|-l\rangle\right)$, phase $\varphi$ represents the position of the pinhole, the coincidence rate is related to the following expression:

$$
C(\theta, \varphi) \propto\left\langle\left.\Phi\right|_{\text {out }} \hat{P} \mid \Phi\right\rangle_{\text {out }}=4 \cos ^{2}\left(\frac{\theta-\varphi}{2}\right)
$$

Equation (12) shows that the coincidence rate is a sinusoidal function of phase $\theta$. The results for $l=1,2$ are shown in Figure $3 \mathrm{j}$ and $3 \mathrm{k}$, where the corresponding visibilities are $89 \% \pm 6 \%$ and $89 \% \pm 7 \%$, respectively, and the error bars are estimated by assuming Poisson statistics for the measured data. Usually, the quality of a quantum transforming process is characterized using fidelity of process. For up-conversion of quantum states, the fidelity is defined as $F=\left\langle\left.\Phi\right|_{\text {in }} \rho_{\text {out }} \mid \Phi\right\rangle_{\text {in }}{ }^{55}$, where $\rho_{\text {out }}=V|\Phi\rangle_{\text {in }}\left\langle\left.\Phi\right|_{\text {in }}+(1-V) / 2\right.$ is the output density matrix and $V$ is the visibility of interference, the fidelity is related to the visibility as $F=(1+V) / 2$. Therefore, the fidelities of up-conversion process for $l=1,2$ are $0.94 \pm 0.03$ and $0.95 \pm 0.04$, respectively.

The effective up-conversion of OAM modes high than $l=2$ are also possible by optimizing the experimental parameters. The feasible methods are: (i) narrowing the spectral bandwidth of the signal photon; (ii) increasing the length of the SFG crystal; (iii) changing the cavity geometric dimension to increase the beam waist inside the crystal in order to support high order spatial mode. The present setup is possible for up-conversion of some simple images at ultra-weak power ( $\mathrm{pW}$ level), such as lower-order OAM modes, OAM superposition mode, and simple spatial shapes with spatial symmetry.

\section{CONCLUSIONS}

We have realized an efficient photonic quantum interface for single photon with both single OAM and OAM superposition. The conversion efficiency for $l=1$ is $8.33 \%$ using our present setup and the coherence properties are retained in the up-conversion process. Also, the detailed theoretical description of OAM-carried light upconversion provides a useful guide for optimizing the conversion process. This primary study will pave the way for high-dimensional quantum information processing in the OAM degree of photons, which create a link between different quantum systems that work in different wavelengths. The present setup can also be possibly used for single-photon-level image up-conversion detection with optimized experimental parameters, which will be of potential importance in many fields, such as biology, astrophysics, night-vision technology, and chemical sensing.

\section{ACKNOWLEDGEMENTS}

The authors would like to thank Dr. Bi-Heng Liu for loaning the InGaAs single photon detectors. This work was supported by the National Fundamental Research Program of China (2011CBA00200), the National Natural Science Foundation of China (11174271, 61275115, and 61435011), and the Innovation Fund from the Chinese Academy of Sciences.

1 Blinov BB, Moehring DL, Duan LM, Monroe C. Observation of entanglement between a single trapped atom and a single photon. Nature 2004; 428: 153-157.

2 Matsukevich DN, Kuzmich A. Quantum state transfer between matter and light. Science 2004; 306: 663-666.

3 Togan E, Chu Y, Trifonov AS, Jiang L, Maze J et al. Quantum entanglement between an optical photon and a solid-state spin qubit. Nature 2010; 466: 730-734.

4 Piro N, Rohde F, Schuck C, Almendros M, Huwer J et al. Heralded single-photon absorption by a single atom. Nat Phys 2011; 7: 17-20.

5 Clausen C, Usmani I, Bussières F, Sangouard N, Afzelius M et al. Quantum storage of photonic entanglement in a crystal. Nature 2011; 469: 508-511.

6 Bussières F, Clausen C, Tiranov A, Korzh B, Verma VB et al. Quantum teleportation from a telecom-wavelength photon to a solid-state quantum memory. Nat Photonics 2014; 8: 775-778.

7 Riedländer D, Kutluer K, Ledingham PM, Gündoğan M, Fekete J et al. Quantum storage of heralded single photons in a Praseodymium-doped crystal. Phys Rev Lett 2014; 112: 040504. 
8 Briegel HJ, Dür W, Cirac JI, Zoller P. Quantum repeaters: the role of imperfect local operations in quantum communication. Phys Rev Lett 1998; 81: 5932-5935.

9 Duan LM, Lukin MD, Cirac JI, Zoller P. Long-distance quantum communication with atomic ensembles and linear optics. Nature 2001; 414: 413-418.

10 Lloyd S, Shahriar MS, Shapiro JH, Hemmer PR. Long distance, unconditiona teleportation of atomic states via complete Bell state measurements. Phys Rev Lett 2001; 87: 167903.

11 Dholakia K, Čižmár T. Shaping the future of manipulation. Nat Photonics 2011; 5: 335-342.

12 Paterson L, Macdonald MP, Arlt J, Sibbett W, Bryant PE et al. Controlled rotation of optically trapped microscopic particles. Science 2001; 292: 912-914.

13 D'Ambrosio V, Spagnolo N, Re LD, Slussarenko S, Li Y et al. Photonic polarization gears for ultra-sensitive angular measurements. Nat Commun 2013; 4: 2432.

14 Lavery MPJ, Speirits FC, Barnett SM, Padgett MJ. Detection of a spinning object using light's orbital angular momentum. Science 2013; 341: 537-540.

15 Zhou ZY, Li Y, Ding DS, Zhang W, Shi S et al. Optical vortex beam based optical fan for high-precision optical measurements and optical switching. Opt Lett 2014; 39: 5098-5101.

16 Wang J, Yang JY, Fazal IM, Ahmed N, Yan Y et al. Terabit free-space data transmission employing orbital angular momentum multiplexing. Nat Photonics 2012; 6: 488496.

17 Bozinovic N, Yue Y, Ren YX, Tur M, Kristensen P et al. Terabit-scale orbital angular momentum mode division multiplexing in fibers. Science 2013; 340: 1545-1548.

18 Mair A, Vaziri A, Weihs G, Zeilinger A. Entanglement of the orbital angular momentum states of photons. Nature 2001; 412: 313-316.

19 Leach J, Jack B, Romero J, Jha AK, Yao AM et al. Quantum correlations in optical angle-orbital angular momentum variables. Science 2010; 329: 662-665.

20 Fickler R, Lapkiewicz R, Plick WN, Krenn M, Schaeff C et al. Quantum entanglement of high angular momenta. Science 2012; 338: 640-643.

21 Dada AC, Leach J, Buller GS, Padgett MJ, Andersson E. Experimental highdimensional two-photon entanglement and violations of generalized Bell inequalities. Nat Phys 2011; 7: 677-680.

22 Barreiro JT, Wei TC, Kwiat PG. Beating the channel capacity limit for linear photonic superdense coding. Nat Phys 2008; 4: $282-286$.

23 Bechmann-Pasquinucci H, Tittel W. Quantum cryptography using larger alphabets. Phys Rev A 2000; 61: 062308.

24 Vallone G, D'Ambrosio V, Sponselli A, Slussarenko S, Marrucci L et al. Free-space quantum key distribution by rotation-invariant twisted photons. Phys Rev Lett 2014 113: 060503.

25 Lauritzen B, Minář J, de Riedmatten H, Afzelius M, Sangouard N et al. Telecommunication-wavelength solid-state memory at the single photon level. Phys Rev Lett 2010; 104: 080502.

26 Saglamyurek E, Jin J, Verma VB, Shaw MD, Marsili F et al. Quantum storage of entangled telecom-wavelength photons in an erbium-doped optical fibre. Nat Photonics 2015; 9: 83-87.

27 Ding DS, Zhou ZY, Shi BS, Guo GC. Single-photon-level quantum image memory based on cold atomic ensembles. Nat Commun 2013; 4: 2527.

28 Nicolas A, Veissier L, Giner L, Giacobino E, Maxein D et al. A quantum memory for orbital angular momentum photonic qubits. Nat Photonics 2014; 8: 234-238.

29 Tanzilli S, Tittel W, Halder M, Alibart O, Baldi P et al. A photonic quantum information interface. Nature 2005; 437: 116-120.

30 Takesue H. Single-photon frequency down-conversion experiment. Phys Rev A 2010; 82: 013833.

31 Curtz N, Thew R, Simon C, Gisin N, Zbinden H. Coherent frequency down-conversion interface for quantum repeaters. Opt Express 2010; 18: 22099-22104.

32 Zaske S, Lenhard A, Keßler CA, Kettler J, Hepp C et al. Visible-to-telecom quantum frequency conversion of light from a single quantum emitter. Phys Rev Lett 2012; 109: 147404

33 Takesue $\mathrm{H}$. Erasing distinguishability using quantum frequency up-conversion. Phys Rev Lett 2008; 101: 173901.

34 Rakher MT, Ma LJ, Slattery O, Tang X, Srinivasan K. Quantum transduction of telecommunications-band single photons from a quantum dot by frequency upconversion. Nat Photonics 2010; 4: 786-791.
35 McGuinness HJ, Raymer MG, McKinstrie CJ, Radic S. Quantum frequency translation of single-photon states in a photonic crystal fiber. Phys Rev Lett2010; 105: 093604.

36 Ates S, Agha I, Gulinatti A, Rech I, Rakher MT et al. Two-photon interference using background-free quantum frequency conversion of single photons emitted by an InAs quantum dot. Phys Rev Lett 2012; 109: 147405

37 Ikuta R, Kusaka Y, Kitano T, Kato H, Yamamoto T et al. Wide-band quantum interface for visible-to-telecommunication wavelength conversion. Nat Commun 2011; 2: 1544.

38 Guerreiro T, Martin A, Sanguinetti B, Pelc JS, Langrock C et al. Nonlinear interaction between single photons. Phys Rev Lett 2014; 113: 173601.

39 Radnaev AG, Dudin YO, Zhao R, Jen HH, Jenkins SD et al. A quantum memory with telecom-wavelength conversion. Nat Phys 2010; 6: 894-899.

40 Dudin YO, Radnaev AG, Zhao R, Blumoff JZ, Kennedy TAB et al. Entanglement of light-shift compensated atomic spin waves with telecom light. Phys Rev Lett 2010; 105: 260502

41 Zhou ZY, Ding DS, Jiang YK, Li Y, Shi S et al. Orbital angular momentum light frequency conversion and interference with quasi-phase matching crystals. Opt Express 2014; 22: 20298-20310.

42 Zhou ZY, Li Y, Ding DS, Zhang W, Shi S et al. Highly efficient second harmonic generation of a light carrying orbital angular momentum in an external cavity. Opt Express 2014; 22: 23673-23678.

43 Li Y, Zhou ZY, Ding DS, Shi BS. Sum frequency generation with two orbital angular momentum carrying laser beams. J Opt Soc Am B 2015; 32: 407-411.

44 Kumar P. Quantum frequency conversion. Opt Lett 1990; 15: 1476-1478.

45 Albota MA, Wong FNC. Efficient single-photon counting at $1.55 \mu \mathrm{m}$ by means of frequency upconversion. Opt Lett 2004; 29: 1449-1451.

46 Beržanskis A, Matijošius A, Piskarskas A, Smilgevičius V, Stabinis A. Conversion of topological charge of optical vortices in a parametric frequency converter. Opt Commun 1997; 140: 273-276.

47 Caetano DP, Almeida MP, Ribeiro PHS, Huguenin JAO, dos Santos BC et al. Conservation of orbital angular momentum in stimulated down-conversion. Phys Rev A 2002; 66: 041801.

48 Molina-Terriza G, Torner L, Minardi S, Trapani PD. Simultaneous frequency conversion and beam shaping for optical-tweezers applications. J Mod Opt 2003; 50: 1563-1572.

49 Devaux F, Passier R. Phase sensitive parametric amplification of optical vortex beams. Eur Phys J D 2007; 42: 133-137.

50 Hansch TW, Couillaud B. Laser frequency stabilization by polarization spectroscopy of a reflecting reference cavity. Opt Commun 1980; 35: 441-444.

51 Kuzmich A, Bowen WP, Boozer AD, Boca A, Chou CW et al. Generation of nonclassical photon pairs for scalable quantum communication with atomic ensembles. Nature 2003; 423: 731-734.

52 Zhou ZY, Jiang YK, Ding DS, Shi BS. An ultra-broadband continuously-tunable polarization entangled photon-pair source covering the $\mathrm{C}+\mathrm{L}$ telecom bands based on a single type-II PPKTP crystal. J Mod Opt 2013; 60: 720-725.

53 Zhou ZY, Jiang YK, Ding DS, Shi BS, Guo GC. Actively switchable nondegenerate polarization-entangled photon-pair distribution in dense wave-division multiplexing. Phys Rev A 2013; 87: 045806.

54 Zhou ZY, Ding DS, Li Y, Wang FY, Shi BS. Cavity-enhanced bright photon pairs at telecom wavelengths with a triple-resonance configuration. J Opt Soc Am B2014; 31: $128-134$.

55 Marcikic I, de Riedmatten H, Tittel W, Zbinden H, Gisin N. Long-distance teleportation of qubits at telecommunication wavelengths. Nature 2003; 421: 509-513.

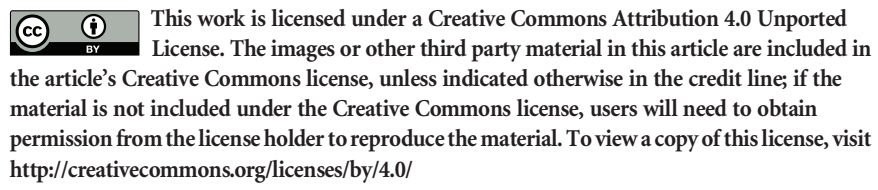

http://creativecommons.org/licenses/by/4.0/ 